Bull. Austral. Math. Soc.

VOL. 37 (1988) [43-59]

\title{
INJECTIVITY AND INJECTIVE HULLS OF ABELIAN GROUPS IN A LOCALIC TOPOS
}

\author{
KIRAN R. BHUTANI
}

We prove the analogue of the Baer Criterion for injectivity in the category $A b S h \mathcal{L}$ of abelian groups in a topos of sheaves on a locale, that is, we show $A$ is injective in $A b S h \mathcal{L}$ if and only if it is injective relative to all $S \mapsto Z_{\mathcal{L}}$ where $Z_{\mathcal{L}}$ is the group of integers in $S h \mathcal{C}$. for a well-ordered locale we describe the injective hulls in $A b S h \mathcal{L}$ in terms of injective hulls in $A b$. Further we show that the global functor $A \rightarrow A E$ preserves injective hulls if and only if $\mathcal{L}$ is a finite boolean locale. Finally we characterise injectives in $A b S h \mathcal{L}$ for some special locales.

\section{INTRODUCTION}

This paper is devoted to the study of injectivity and injective hulls in the category $A b S h \mathcal{L}$ of abelian groups in a topos of sheaves on a local $\mathcal{L}$. We first prove the analogue of the Baer criterion for injectivity in $A b S h \mathcal{L}$ (Proposition 1.1) and show that injectivity is a local property (1.4). This is followed by a discussion on injective hulls, which we show is a local property (Proposition 2.1) but not a global one. For a well-ordered locale we describe the injective hulls in $A b S h \mathcal{L}$ in terms of injective hulls in $A b$ (Proposition 2.3). Further, in Proposition 2.7 we show that the global functor preserves injective hulls if and only if $\mathcal{L}$ is a finite boolean locale, that is, the topologies of finite discrete spaces.

Finally, we characterise in Propositions 3.1 and 3.3, the injectives in $A b S h \mathcal{L}$ for the following locales:

1) $\mathcal{L}$ with descending chain condition

2) $\mathcal{L}$ inversly well ordered

As a consequence we show that the direct sum of injectives in $A b S h \mathcal{L}$ is always injective for $\mathcal{L}$ inversely well ordered (Corollary 3.4). This does not hold for an arbitary $\mathcal{L}$ and a counter example is provided (3.5).

In Section 0 we describe briefly the background material required here, where as in Section 1 we derive general results on injectivity. In Section 2 we discuss injective hulls and finally we characterise injectives for some special locales in Section 3 . The $n^{\text {th }}$ result in the $m^{\text {th }}$ Section will be numbered as $m . n$.

Received 4 March 1987

Abstracted partially from the dissertation submitted March 1983 to McMaster University for partial fulfillment of the requirements for the degree of Doctor of Philosophy. The author is grateful to her supervisor Professor Bernhard Banaschewski, for his valuable assistance, encouragement and criticism throughout this work. Facilities for the preparation of this manuscript were provided by the Division of Computer Research and Technology at the National Institutes of Health, Bethesa, MD.

Copyright Clearance Centre, Inc. Serial-fee code: 0004-9729/88 \$A2.00+0.00. 


\section{BACKGROUND}

DEFINITION 0.1: Recall that a locale denote by $\mathcal{L}$ is a complete lattice satisfying the following;

$$
U \wedge \bigvee_{i \in I} U_{i}=\bigvee_{i \in I}\left(U \wedge U_{i}\right)
$$

for all $U$ and any family $\left\{U_{i}\right\}_{i \in I}$ in $\mathcal{L}$. We shall denote the bottom (=zero) of $\mathcal{L}$ by 0 and the top (=unit) of $\mathcal{L}$ by $E$. A morphism of locales $h: \mathcal{L} \rightarrow \mathcal{M}$ is a map which preserves arbitary joins and finite meets (hence preserves the zero and the unit). The obvious example of a locale is the topology $\mathcal{O} X$ (that is, the lattice of open sets) of any topological space $X$ with joins as unions and meets as intersections. Other examples of locales are a complete chain, complete boolean algebra or a finite distributive lattice.

By the definition of continuity of maps between topological spaces, we get a contravariant functor $\mathcal{O}: T O P \rightarrow L O C$ where $T O P$ is the category of topological spaces and continuous maps, and $L O C$ is the category of locales and their morphisms. The functor $\mathcal{O}$ has an adjoint on the right, the contravariant functor $\Sigma: L O C \rightarrow$ TOP where $\Sigma \mathcal{L}$ is the space of completely prime filters $F$ on $\mathcal{L}$, that is, $F$ is a filter on $\mathcal{L}$ such that $\bigvee_{i \in I} U_{i} \in F$ for any family $\left\{U_{i}\right\}_{i \in I}$ in $\mathcal{L}$ implies $U_{k} \in F$ for some $k \in I$, and the sets $\Sigma_{U}=\{F \mid U \in F, F \in \Sigma \mathcal{L}\}, U \in \mathcal{L}$, form the open sets in this space. For any locale lattice homomorphism $h: \mathcal{L} \rightarrow \mathcal{M}$ the corresponding continuous map $\Sigma h: \Sigma \mathcal{M} \rightarrow \Sigma \mathcal{L}$ sends $F \rightarrow h^{-1}(F)$. The space $\Sigma \mathcal{L}$ is called the spectrum of $\mathcal{L}$.

DEFinition 0.2: A locale $\mathcal{L}$ is called spatial if and only if the function $\mathcal{L} \rightarrow \mathcal{O}(\Sigma \mathcal{L})$ is an isomorphism. Since $\mathcal{O}_{\mathcal{L}}$ is always onto, a locale is spatial if and only if the completely prime filters separate points in $\mathcal{L}$. For more details refer to [4]. Note that any finite locale is spatial since in any distributive lattice the prime filters separate the elements (Balbes and Dwinger [1]), and for finite $\mathcal{L}$ the prime filters are completely prime. Also any totally ordered locale is spatial since the $U \geqslant V, V \in \mathcal{L}$ form a completely prime filter on $\mathcal{L}$ for any $U \in \mathcal{L}$.

Finally any $\mathcal{L}$ with descending chain condition is spatial: If $U \leqslant V$ in $\mathcal{L}$ and $W \in \mathcal{L}$ is minimal such that $U<W \leqslant V$ then $F=\{S \mid S \in \mathcal{L}, U \vee S \geqslant W\}$ is a completely prime filter on $\mathcal{L}$ for which $U \notin F$ but $V \in F$.

Definition 0.3: A locale $\mathcal{L}$ is boolean if and only if every element in $\mathcal{L}$ has a complement. This is equivalent to saying that $\mathcal{L}$ has no dense elements other than $E$. That is, there is no $W \neq E$ such that $U \wedge W=0$ implies $U=0$. Note that a boolean locale is spatial if and only if $\mathcal{L}$ is atomic, [1], the non-trivial implication follows since any completely prime filter in a boolean locale is a principal filter given by an atom.

Definition 0.4: By $A b S h \mathcal{L}$ and $A b P S h \mathcal{L}$ we mean the categories of Sheaves and Presheaves on $\mathcal{C}$ with values in the category $A b$ of Abelian groups. These are 
Grothendieck categories with generator and hence have enough injective hulls [12]. $A b S h \mathcal{L}$ forms a full subcategory of $A b P S h \mathcal{L}$ and the inclusion $A b S h \mathcal{L} \rightarrow A b P S h \mathcal{L}$ has an exact left adjoint the sheaf reflection functor $A b P S h \mathcal{L} \rightarrow A b S h \mathcal{L}$. If $A$ is the sheaf reflection of a given presheaf $B$, then we shall write $A U \doteq B U, U \in \mathcal{L}$. If $\mathcal{L}=\mathcal{O}(X)$ for some topological space $X$, then we shall write $A b S h X$ for $A b S h O(X)$. Also for any map $h: A \rightarrow B$ in $A b S h \mathcal{L}$ (and hence also in $A b P S h \mathcal{L}$ ) $h_{U}: A U \rightarrow B U$ will be the component of $h$ at $U \in \mathcal{L}$. For any $a \in A U$ and $W \leqslant U$, the map $A U \rightarrow A W$ will be denoted $a \longrightarrow a \mid W$.

REMARK 0.5: $A b S h 2 \cong A b$ for the two element locale 2 , and if $X$ is a discrete topological space then $A b S h X \cong A b^{|X|}$.

Definition 0.6: Any local lattice homomorphism $\phi: \mathcal{L} \rightarrow \mathcal{M}$ produces a pair of adjoint functors $A b S h \mathcal{M} \stackrel{\phi_{*}}{\longrightarrow} A b S h \mathcal{L}$ where $\left(\phi_{*} A\right) U=A(\phi(U))$, and for any $V \in \mathcal{M}\left(\phi^{*} C\right) V \doteq \stackrel{\ell t}{\longrightarrow}_{\phi(W) \geqslant V} C W(W \in \mathcal{L})$. Then $\phi^{*}$ is left exact left adjoint to $\phi_{*}$ and from well-known results it follows that $\phi_{*}$ preserves injectives.

Definition 0.7: Special cases of local lattice homomorphisms.

1) If $\phi: 2 \rightarrow \mathcal{L}$ is the unique local lattice homomorphism, then it gives $A b S h \mathcal{L} \rightarrow$ $A b S h 2 \cong A b$, where $\left(\phi_{*} A\right)=A E$ and $\left(\phi^{*} B\right) U \doteq B$. Notation $\phi^{*} B=B_{\mathcal{C}}, \phi_{*}=\Gamma$.

2) Any local lattice homomorphism $\phi: \mathcal{L} \rightarrow 2$ produces $A b \rightarrow A b S h \mathcal{L}$ where

$$
\left(\phi_{*} A\right) U= \begin{cases}A & \text { if } \phi(U)=1 \\ 0 & \text { if } \phi(U)=0\end{cases}
$$

and $\phi^{*} A=\ell t A U$ (All $U$ such that $\phi(U)=1$ ).

3) If $\mathcal{L}=\mathcal{O}(X)$ for some topological space $X$, and $x \in X$ is any point, then for the local lattice homomorphism $\hat{x}: \mathcal{L} \rightarrow 2$ given by $\hat{x}(U)=\operatorname{card}(U \cap\{x\})$, we get $(\hat{x})^{*} A=\stackrel{\text { et }}{\longrightarrow} A U(x \in U)=A_{x}$, the stalk of $A$ at $x$.

4) For any $U \in \mathcal{L}, \phi: \mathcal{L} \rightarrow \downarrow U$ given by $\phi(W)=W \wedge U$ is a local lattice $\stackrel{\text { 中. }}{\longrightarrow}$

homomorphism, so we get $A b S h \downarrow U \underbrace{U}_{\phi^{*}} A b S h \mathcal{L}$, where $\left(\phi_{*} A\right) V=A(V \wedge U)$ and $\left(\phi^{*} B\right) W \doteq \stackrel{\ell t}{\longrightarrow}_{\phi(V) \geqslant W} B V=B W$, and so $\phi^{*} B$ is just the restriction of $B$ to $\downarrow U$.

Notation : $\phi^{*} B=B \mid U=R_{U} B$.

Also $\phi^{*}$ has a left adjoint denoted by $E_{U}$, where

$$
\left(E_{U} A\right) V \doteq \begin{cases}A V & \text { if } V \leqslant U \\ 0 & \text { if } V \notin U .\end{cases}
$$


Then $E_{U}$ is left exact left adjoint to $R_{U}$. Since $R_{U}$ is both a right adjoint as well as a left adjoint, it preserves all limits and colimits.

5) If $f: X \rightarrow Y$ is a continuous map of topological spaces, then it produces a local lattice homomorphism (also denoted by $f$ ) $f: \mathcal{O} Y \rightarrow \mathcal{O} X, V \rightarrow f^{-1}(V)$, and so correspondingly it gives $A b S h X \rightarrow A b S h Y$. In particular for any topological space $X$, let $|X|$ be $X$ with discrete topology. Then the identity map $i:|X| \rightarrow X$ is continuous, hence it produces $A b^{|X|} \cong A b S h|X| \rightarrow A b S h X$.

Definition 0.8: $A \in A b S h \mathcal{L}$ is said to be a divisible group if for any $a \in A U$, and any $O \neq n \in N$ there exists a cover $U=\bigvee_{i \in I} U_{i}$ in $\mathcal{L}$, such that for all $i \in I$, $a \mid U_{i}=n b_{i}$ with $b_{i} \in A U_{i}$.

Definition 0.9: For any $A \in A b S h \mathcal{L}$ the subgroup $C$ of $A$ generated by an element $a \in A U, U \in \mathcal{L}$, that is, the smallest subgroup $C \subseteq A$ such that $a \in C U$, is given by

$$
C W \doteq \begin{cases}Z(a \mid W) & \text { if } W \subseteq U \\ 0 & \text { if } W \nsubseteq U\end{cases}
$$

Definition 0.10: $B \supseteq A$ is an essential extension in $A b S h \mathcal{L}$ if and only if for any $0 \neq b \in B U$, there exists $V \leqslant U$ in $\mathcal{L}$ and $m \in Z$ such that $0 \neq m b \mid V \in A V$. To see this, one first notices that $B \supseteq A$ is essential if and only if $C \cap A \neq 0$ for any non zero subgroup $C \subseteq B$ (since a homomorphism in $A b S h \mathcal{L}$ is monic if and only if its kernel is 0 ), and then observe that it is sufficient to consider subgroups generated by a single non-zero $b \in B U$ for any $U \in \mathcal{L}$.

Proposition 0.11. For any $U \in \mathcal{L}$, the functors $R_{U}: A b S h \mathcal{C} \rightarrow A b S h \downarrow U$, and $E_{U}: A b S h \downarrow U \rightarrow A b S h \mathcal{L}$ preserve essential extensions.

Proof: Consider any essential extension $B \supseteq A$ in $A b S h \mathcal{L}$. Since $R_{U}$ preserves all limits $(0.7(4))$, it follows that $B|U \supseteq A| U$. We claim this is an essential extension in $A b S h \downarrow U$. Let $0 \neq b \in B W=(B \mid U)(W)$ for some $W \in \downarrow U$. Since $B$ is an essential extension of $A$ in $A b S h \mathcal{L}$, there exists a $V \leqslant W, m \in Z$ such that $0 \neq m b \mid V \in A V=(A \mid U)(V)$. Hence $B|U \supseteq A| U$ is an essential extension in $A b S h \downarrow U$. To prove that $E_{U}$ preserves essential extensions, consider an essential extension $P \supseteq Q$ in $A b S h \downarrow U$. Since $E_{U}$ preserves monomorphisms $(0.7(4))$ it follows that $E_{U} P \supseteq E_{U} Q$. Let $0 \neq a \in\left(E_{U} P\right) W$, then by the definition of $E_{U}$, there exists a cover $W=\bigvee_{i \in I} W_{i}$, such that $0 \neq a \mid W_{i} \in P W_{i}$ for some $W_{i} \subseteq U$. But $P \supseteq Q$ is an essential extension in $A b S h \downarrow U$ so there exists $V \leqslant W_{i}$ and an $m \in Z$ such that $0 \neq m\left(a \mid W_{i}\right)|V=m a| V \in Q V$. Hence $E_{U} P \supseteq E_{U} Q$ is an essential extension in $A b S h \mathcal{L}$.

Corollary 0.12. For any $U \in \mathcal{L}, R_{U}$ preserves injectives and injective hulls. 
Proof: Since $R_{U}$ has a left adjoint $E_{U}$ which preserves monomorphisms $(0.8(4))$, it follows that $R_{U}$ preserves injectives. By the above proposition if follows $R_{U}$ preserves injective hulls.

Corollary 0.13. For an injective group $A \in A b S h \mathcal{L}, A U$ is an injective group in $A b$ for all $U \in \mathcal{L}$.

Proof: Clear from 0.12 and $0.7(1)$.

Remark 0.14: The composition $E_{U} R_{U}$ is denoted by $T_{U}: A b S h \mathcal{L} \rightarrow A b S h \mathcal{L}$, where

$$
\left(T_{U} A\right) W \doteq \begin{cases}A W & \text { if } W \subseteq U \\ 0 & \text { if } W \nsubseteq U\end{cases}
$$

Since both $E_{U}$ and $R_{U}$ preserve essential extensions, it follows that $T_{U}$ preserves essential extensions. Note, though, that $T_{U}$ does not preserve injectives, as one can see by considering $\mathcal{L}=3$.

REMARK 0.15: It is easily checked that $A b S h \mathcal{L}$ has the $T_{U} Z_{\mathcal{L}}, U \in \mathcal{L}$, as generating set where $Z_{\mathcal{L}}$ is the group of integers in $S h \mathcal{L}$, that is, the sheaf reflection of the constant presheaf $Z$.

\section{General Results}

\section{The Baen Criterion for INJectivity}

Proposition 1.1. $A \in A b S h \mathcal{L}$ is injective if and only if it in injective relative to all $S \mapsto Z_{\mathcal{L}}$.

Proof: $(\Rightarrow)$ is trivial.

$(\Leftarrow)$ Let $A$ be injective relative to all $S \mapsto Z_{\mathcal{L}}$. Consider the diagram

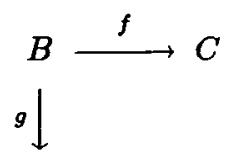

A

where we may assume that $B \subseteq C$, and $f: B \mapsto C$ is the natural embedding. Consider the family $\mathcal{A}=\left\{\left(B^{\prime}, g^{\prime}\right)\right\}$ where $B \subseteq B^{\prime} \subseteq C$ and $g^{\prime}: B^{\prime} \rightarrow A$ such that $g^{\prime} \mid B=g$. Then this family is non-empty since $(B, g) \in \mathcal{A}$. As usual, we introduce a partial ordering on this family by $\left(B^{\prime}, g^{\prime}\right) \leqslant\left(B^{\prime \prime}, g^{\prime \prime}\right)$ if and only if $B^{\prime} \subseteq B^{\prime \prime}$ and $g^{\prime \prime} \mid B^{\prime}=g^{\prime}$. If $\left\{\left(B_{i}, g_{i}\right)\right\}_{i \in I}$ is a linearly ordered family in $\mathcal{A}$, then it has an upper bound in $\mathcal{A}$ given by $(D, h)$, where $D$ is the join of $B_{i}$ in the subgroup lattice of $C$. That is, $D$ is the sheaf reflection of the presheaf $U \rightarrow \bigcup_{i \in I} B_{i} U$ and $h$ is the corresponding sheaf reflection of the morphism to which the $g_{i_{U}}$ extend. By Zorn's lemma, the family $\mathcal{A}$ 
has a maximal element $(P, p)$. We claim $P=C$. If not, then there is a $U \in \mathcal{L}$ and $c \in C U$ such that $c \notin P U$. Let $H$ be the subgroup of $C$ generated by $c$. Then $H$ is the sheaf reflection of the presheaf

$$
W \rightarrow \begin{cases}Z(c \mid W) & \text { if } W \subseteq U \\ 0 & \text { if } W \nsubseteq U\end{cases}
$$

Since the presheaf defining $T_{U} Z_{\mathcal{L}}$ is given by $W \rightarrow\left\{\begin{array}{ll}Z & \text { if } W \subseteq U \\ 0 & \text { if } W \nsubseteq U\end{array}\right.$ therefore, there is an epimorphism of presheaves from that defining $T_{U} Z_{\mathcal{L}}$ to that defining $H$. The sheaf reflection preserves epimorphisms, and so $j: T_{U} Z_{\mathcal{L}} \rightarrow H$ is an epimorphism in $A b S h \mathcal{L}$. The diagram

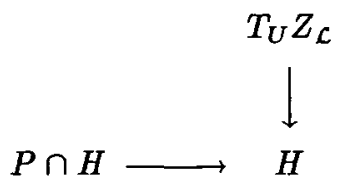

can be completed to a pull-back square,

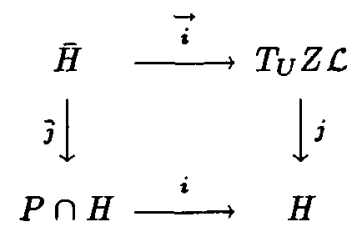

where $\bar{\imath}$ is a mono since $i$ is. Moreover since $j$ is an epimorphism, $\bar{j}$ is an epimorphism, and the above diagram is actually a push out diagram [16, p.33]. But $T_{U} Z_{\mathcal{L}} \subseteq Z_{\mathcal{L}}[\mathbf{5}]$, and $A$ is injective relative to all $\bar{H} \rightarrow Z_{\mathcal{L}}$, so there exists $\alpha: Z_{\mathcal{L}} \rightarrow A$ such that the outer triangle of the diagram

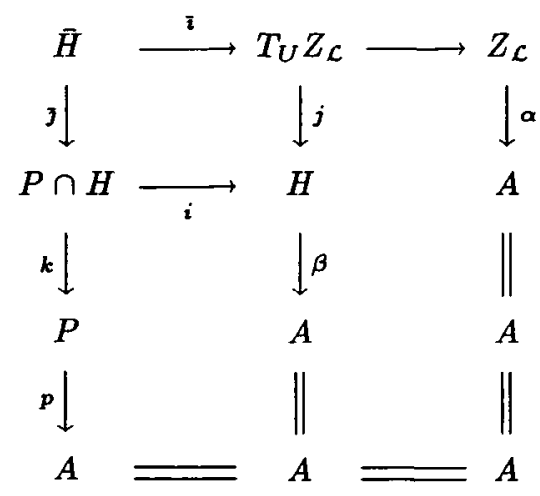


commutes; that is, $\left(\alpha \mid T_{U} Z_{\mathcal{L}}\right) \bar{\imath}=p k \bar{\jmath}$. The inner square is also a push out square, and so there exists a unique $\beta: H \rightarrow A$ such that $\beta j=\alpha \mid T_{U} Z_{\mathcal{L}}$ and $\beta i=p k$. Define another presheaf $M$ by $M U=P U+H U \subseteq C U$, with the obvious restriction maps, then

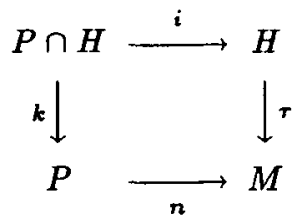

is a push-out in $A b S h \mathcal{L}$, because at each $U \in \mathcal{L}$ it is a push-out in $A b$. Since $P+H \doteq$ $M$, and the sheaf reflection being a left adjoint preserves push out, it follows that

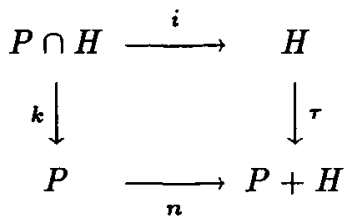

is a push-out in $A b S h \mathcal{L}$. Hence if we consider the diagram

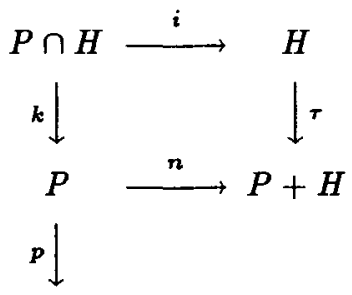

A

then there is a unique $q: P+H \rightarrow A$ such that $q n=p$, and $q \tau=\beta$. Thus $(P+H, q) \in$ $\mathcal{A}$, a contradiction, since $(P, p) \in \mathcal{A}$ is maximal and $P+H \supset P$. Thus $P=C$, and hence $A$ is injective.

REMARK: Although the analogue of the Baer Criterion for injectivity holds in $A b S h \mathcal{L}$, still the concepts of injectivity and divisibility do not coincide for an arbitary $\mathcal{L}$. In fact the two concepts coincide if and only if $\mathcal{L}$ is Boolean [2].

LEMma 1.2. For any cover $E=\bigvee_{i \in I} U_{i}$ of the unit in $\mathcal{C}$, the functor $R: A b S h \mathcal{L}$ $\rightarrow \prod_{i \in I} A b S h \downarrow U_{i}$ given by $R B=\left(B \mid U_{i}\right)_{i \in I}, R h=\left(h \mid U_{i}\right)_{i \in I}$ for $h: A \rightarrow B$ in $A b S h \mathcal{L}$, has the following two properties:

(a) $R$ preserves and reflects monomorphisms.

(b) $R$ is faithful.

Proof: (a) If $h: A \rightarrow B$ is a monomorphism in $A b S h \mathcal{L}$, then each $h\left|U_{i}: A\right|$ $U_{i} \rightarrow B \mid U_{i}$ is a monomorphism in $A b S h \downarrow U_{i}(0.7(4))$, hence $\left(h \mid U_{i}\right): R A \rightarrow R B$ is a 
monomorphism in $\prod_{i \in I} A b S h \downarrow U_{i}$. Now suppose $R h$ is a monomorphism; we want to show that $h$ is a monomorphism. Let $W \in \mathcal{L}$ be arbitary, and suppose $h_{W}(a)=h_{W}(b)$ for some $a, b \in A W$. Since $h$ is a morphism of sheaves, therefore

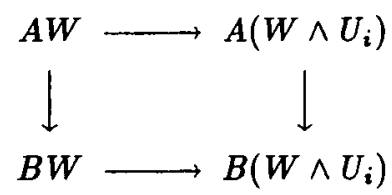

commutes for all $i \in I$, and hence $h_{W \wedge U_{i}}\left(a \mid W \wedge U_{i}\right)=h_{W \wedge U_{i}}\left(b \mid W \wedge W_{i}\right)$. But $h_{W \wedge U_{i}}$ is a monomorphism in $A b S h \downarrow U_{i}$ for all $i$, and therefore $a\left|W \wedge U_{i}=b\right| W \wedge U_{i}$ all $i$, which by the sheaf properties of $A$ implies $a=b$. Hence $h$ is a monomorphism. Thus $R$ preserves and reflects monomorphisms.

(b) Suppose $R f=R g$ for some $f, g: A \rightarrow B$ in $A b S h \mathcal{L}$. Then $f\left|U_{i}=g\right| U_{i}$ for all $i \in I$. We claim $f=g$, that is $f_{W}=g_{W}$ for all $W \in \mathcal{L}$, For any $a \in A W$, we have $g_{W}(a)\left|W \wedge U_{i}=g_{W \wedge U_{i}}\left(a \mid W \wedge U_{i}\right)=f_{W \wedge U_{i}}\left(a \mid W \wedge U_{i}\right)=f_{W}(a)\right| W \wedge U_{i}$, all $i \in I$. Thus for the cover $W=\bigvee_{i \in I} W \wedge U_{i}$, we have $f_{W}(a)\left|W \wedge U_{i}=g_{W}(a)\right| W \wedge U_{i}$, all $i \in I$, hence $f_{W}(a)=g_{W}(a)$. Thus $f_{W}=g_{W}$ for all $W \in \mathcal{L}$ implies $f=g$.

Proposirion 1.3. The functor $R$ preserves and reflects injectives.

Proof: If $B$ is injective in $A b S h \mathcal{L}$, then each $B \mid U_{i}$ is injective in $A b S h \downarrow U_{i}$ (0.12), hence $R B=\left(B \mid U_{i}\right)_{i \in I}$ is injective in $\prod_{i \in I} A b S h \downarrow U_{i}$.

Assume $R B$ is injective; we want to show that $B$ is injective. Consider an essential extension $D$ of $B$. Since $R_{U_{i}}$ preserves essential extensions $(0.11)$ it follows that each $D\left|U_{i} \supseteq B\right| U_{i}$ is an essential extension in $A b S h \downarrow U_{i}$. So if $0 \neq S \subseteq R D$ then for some $i \in I, 0 \neq S_{i} \subseteq D \mid U_{i}$, hence $S_{i} \cap B \mid U_{i} \neq 0$. This means $S \cap R B \neq 0$ which shows $R D$ is an essential extension of $R B$. But $R B$ is given to be injective, and so $R B=R D$. Since $R$ is faithful it reflects epimorphisms, hence the natural embedding $B \rightarrow D$ is an epimorphism and therefore $B=D$. Thus $B$ has no proper essential extensions in $A b S h \mathcal{L}$, which means $B$ is injective (0.4), hence the result.

Propositron 1.4. $B$ is injective in $A b S h \mathcal{L}$ if and only if there is a cover $E=$ $\bigvee_{i \in I} U_{i}$ such that $B \mid U_{i}$ is injective in $A b S h \downarrow U_{i}$, for all $i \in I$.

Proof: $(\Rightarrow)$ Clear by taking the trivial cover of $E$.

$(\Leftrightarrow)$ For the converse assume $E=\bigvee U_{i}$ and each $B \mid U_{i}$ is injective. Then let $A \supseteq B$ be any essential extension. By 0.11 one then has an essential extension $A\left|U_{i} \supseteq B\right| U_{i}$, hence by hypothesis $A\left|U_{i}=B\right| U_{i}$ and then, finally $A=B$, showing that $B$ is injective.

REMARK: The above proposition shows injectivity is a local property. This was also shown by Harting [11], but by an entirely different method. She considers the 
preservation of maximal partial morphisms by the restriction functors $R_{U}$ for $U \in \mathcal{L}$, whereas our approach uses the preservation of essentialness by the functors $R_{U}$.

Lemma 1.5. If $A$ is injective in $A b S h \mathcal{L}$, then for any $V \leqslant U$ in $\mathcal{L}$ the restriction $A U \rightarrow A V$ is a split epimorphism in $A b$.

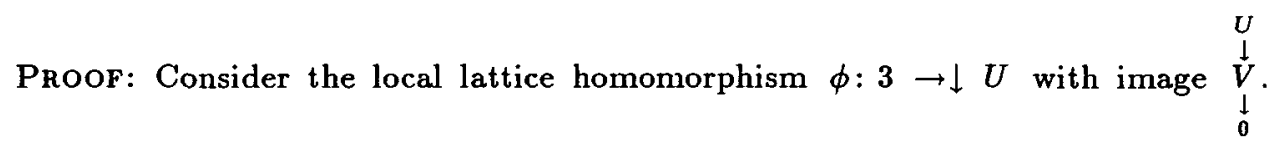
Then since $A$ is injective in $A b S h \mathcal{L}$, it follows that $\phi_{*} A=\underset{A V}{A U}$ is an injective group in $A b S h 3$ (0.6). But the injectives in $A b S h 3$ are exactly the projections $\underset{P}{P \times T}$ with divisible $P$ and $T[2]$, hence $\underset{A V}{A}$ is a split epinorphism in $A b$.

\section{INJECTIVE HULLS}

Given $A, B$ in $A b S h \mathcal{L}$, recall that $B$ is the injective hull of $A$ if and only if it is an essential injective extension of $A$.

Proposition 2.1. $B$ is the injective hull of $A$ in $A b S h \mathcal{L}$, if and only if there exists a cover $E=\bigvee_{i \in I} U_{i}$, such that $B \mid U_{i}$ is the injective hull of $A \mid U_{i}$ in $A b S h \downarrow U_{i}$.

Proof: $(\Rightarrow)$ Clear, by taking the trivial cover.

$(\Leftarrow)$ Given that $B \mid U_{i}$ is the injective hull of $A \mid U_{i}$, all $i \in I$, it follows by 1.4 that $B$ is injective in $A b S h \mathcal{L}$. So, it only remains to show that $B$ is an essential extension of $A$. Let $D \subseteq B$ be a non zero subgroup of $B$, then $D U \neq 0$ for some $U \in \mathcal{L}$. Since $U=\bigvee_{I}\left(U \wedge U_{i}\right)$, it follows that $0 \neq D U \mapsto \prod D\left(U \wedge U_{i}\right)$ and so for some $i \in I$, $0 \neq D\left(U \wedge U_{i}\right)=\left(D \mid U_{i}\right)\left(U \wedge U_{i}\right)$. But $B \mid U_{i}$ is an essential extension of $A \mid U_{i}$ in $A b S h \downarrow U_{i}$, so $0 \neq D\left|U_{i} \subseteq B\right| U_{i}$ implies $D\left|U_{i} \cap A\right| U_{i} \neq 0$, and therefore $D \cap A \neq 0$. Hence $B$ is an essential extension of $A$, and also being injective it is the injective hull of $A$.

REMARK 2.2: In our next result, we describe the injective hull of any $A$ in $A b S h \mathcal{L}$ where $\mathcal{L}$ is well-ordered, and so it might be appropriate to describe the topology of the spectrum of a well-ordered locale. If $\mathcal{L}$ is well-ordered, then without loss of generality we may assume $\mathcal{L}=\lambda+1$, for some ordinal $\lambda$. We now show that the sets $W_{\alpha}=\{\gamma$ : $\gamma$ not a limit ordinal, $0<\gamma \leqslant \alpha\}$ for each $\alpha \in \lambda+1$, form a topology $\mathcal{O}$ on the set $X$ consisting of all the non-zero non-limit ordinals $\gamma \leqslant \lambda$. Now $W_{0}=\emptyset, W_{\lambda}=|X|$, $W_{\alpha} \cap W_{\beta}=W_{\alpha \wedge \beta}$ since for $\alpha \leqslant \beta, W_{\alpha} \subseteq W_{\beta}$. To check $W_{\bigvee_{I} \alpha_{i}}=\bigcup_{I} W_{\alpha_{i}}$ for any family $\left\{\alpha_{i}\right\}_{I}$ in $\lambda+1$, we consider $\gamma \in W_{V_{I} \alpha_{i}}$. Then $\gamma \leqslant V_{I} \alpha_{i}$, so if $\gamma \notin \bigcup_{I} W_{\alpha_{i}}$ then we must have $\alpha_{i}<\gamma$, for all $i \in I$. But $\gamma \in X$ and so $\gamma=\beta+1$ for some 
$\beta<\lambda$. Therefore $\alpha_{i}<\gamma$ implies $a_{i} \leqslant \beta$ for all $i \in I$, hence $\bigvee_{I} \alpha_{i} \leqslant \beta<\gamma$ a contradiction, since $\gamma \leqslant V_{I} \alpha_{i}$. Thus there is some $i \in I$ such that $\gamma \leqslant \alpha_{i}$ and so $\gamma \in \bigcup_{I} W_{\alpha_{i}}$. Therefore $W_{V_{i}} \alpha_{i} \subseteq \bigcup_{I} W_{\alpha_{i}}$. Moreover for all $i \in I, \alpha_{i} \leqslant V_{I} \alpha_{i}$ implies $\bigcup W_{\alpha_{i}} \subseteq W_{V_{I} \alpha_{i}}$ and hence $W_{V_{i} \alpha_{i}}=\bigcup_{I} W_{\alpha_{i}}$. Therefore $\mathcal{O}$ is indeed a topology on $X$. Now let $W_{\alpha}=W_{\beta}$ for some $\alpha, \beta \in \lambda+1$, and suppose $\alpha<\beta$. Then $\alpha+1 \leqslant \beta$ and so $\alpha+1 \in W_{\beta}=W_{\alpha}$ which means $\alpha+1 \leqslant \alpha$, a contradiction. Hence $W_{\alpha}=W_{\beta}$ implies $\alpha=\beta$. Therefore $\mathcal{L}=\lambda+1$ is isomorphic to $\mathcal{O}$ by $\alpha \rightarrow W_{\alpha}$. Note that the completely prime filters on $\mathcal{L}=\lambda+1$ are exactly $\uparrow \gamma$ for $\gamma \in X$, hence $\sum \mathcal{L}$ may be represented by the set $X$ of these $\gamma$ with the topology $W_{\alpha}=\{\gamma \in X \mid \gamma \leqslant \alpha\}$.

Proposition 2.3. For a well-ordered locale $\mathcal{L}$, the injective hull of any $A=$ $A_{\lambda} \stackrel{h_{\gamma}}{\longrightarrow} \cdots \rightarrow A_{2} \stackrel{h_{2}}{\longrightarrow} A_{1} \stackrel{h_{1}}{\longrightarrow} A_{0}(=0)$ in $A b S h \mathcal{L}$ is given by the group $C=C_{\lambda} \rightarrow$ $\cdots \rightarrow C_{2} \rightarrow C_{1} \rightarrow C_{0}(=0)$ where $C_{\beta}=C W_{\beta}=\prod_{\alpha \in W_{\beta}} E\left(\operatorname{Ker} h_{\alpha}\right)$ for all $\beta \in \lambda+1$.

Proof: Define a family $\left(B_{\alpha}\right)_{\alpha \in|X|}$ in $A b^{|X|}$ by $B_{\alpha}=E\left(\operatorname{Ker} h_{\alpha}\right)$ for all $\alpha \in$ $X$. Since the functor $F: A b^{|X|} \rightarrow A b S h X \cong A b S h \mathcal{L}$ preserves injectives $(0.6,0.7)$ it produces an injective $C$ in $A b S h \mathcal{C}$, where $C=F\left(\left(B_{\alpha}\right)_{\alpha \in|X|}\right)$, and so $C_{\beta}=C W_{\beta}=$ $\prod_{\alpha \in W_{\beta}} E\left(\operatorname{Ker} h_{\alpha}\right), \beta \in \lambda+1$ with restrictions $C U_{\beta} \rightarrow C U_{\gamma}$ as projections for all $\gamma \leqslant \beta$. The morphism from $A$ to $C$ is obtained by induction as follows: For $n=1$, $A_{1} \rightarrow C_{1}=E\left(\operatorname{Ker} h_{1}\right)=E\left(A_{1}\right)$ is the natural embedding. Assume $A_{\alpha} \rightarrow C_{\alpha}$ already defined for all $\alpha<\beta$. Then there are two possibilities:

Case (i) $\beta=\gamma+1$ for some $\gamma \in \lambda+1$;

Case (ii) $\beta$ is a limit ordinal.

For case (i) we are given $A_{\gamma} \rightarrow C_{\gamma}$ and since $C_{\gamma+1}=C_{\gamma} \times E\left(\operatorname{Ker} h_{\gamma+1}\right)$ with $C_{\gamma+1} \rightarrow C_{\gamma}$ the projection, we can define $\tau_{\gamma+1}: A_{\gamma+1} \rightarrow C_{\gamma} \prod E\left(\right.$ Ker $\left.h_{\gamma+1}\right)$ as $\tau_{\gamma} h_{\gamma+1} \prod h \frac{}{\gamma+1}$ where $h_{\gamma+1}: A_{\gamma+1} \rightarrow E\left(\operatorname{Ker} h_{\gamma+1}\right)$ is an extension of the natural embedding $\operatorname{Ker}\left(h_{\gamma+1}\right) \rightarrow E\left(\operatorname{Ker} h_{\gamma+1}\right)$ to $A_{\gamma+1}$ : then as required $P_{\gamma} \tau_{\gamma+1}=$ $p_{\gamma}\left(\tau_{\gamma} h_{\gamma+1} \Pi h \frac{}{\gamma+1}\right)=\tau_{\gamma} h_{\gamma+1}$; that is,

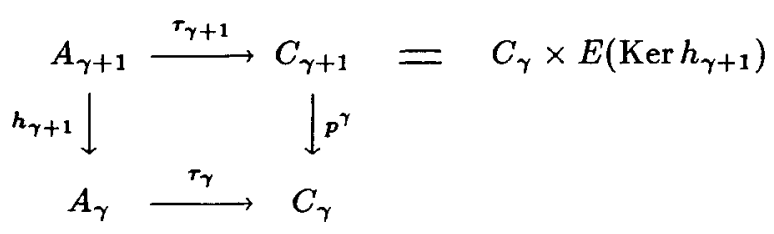

commutes.

Case (ii) $\beta=\bigvee_{\alpha<\beta} \alpha$, and so $C_{\beta}=\underset{\alpha<\beta}{\ell t} C_{\alpha}$. Since $A_{\beta}=\underset{\alpha<\beta}{\ell t} A_{\alpha}$, and by 
assumption all $A_{\alpha} \stackrel{r_{\alpha}}{\longrightarrow} C_{\alpha}(\alpha<\beta)$ are defined, therefore we get a family of maps $A_{\beta} \rightarrow A_{\alpha} \stackrel{r_{\alpha}}{\longrightarrow} C_{\alpha}(\alpha<\beta)$ and so by the definition of limit there is a unique $\tau_{\beta}: A_{\beta} \rightarrow C_{\beta}$ such that

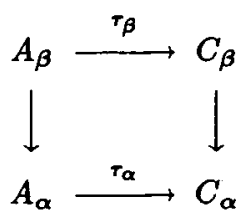

commutes for all $\alpha<\beta$. Hence we can define a morphism $\tau: A \rightarrow C$ with components $\tau_{\alpha}: A_{\alpha} \rightarrow C_{\alpha}$ as defined above.

Now to check that $\tau$ is a monomorphism. Clearly $\tau_{1}$, is a monomorphism, so assume $\tau_{\alpha}$ is a mono for all $\alpha<\beta$.

Case (i) $\beta=\gamma+1$, so if $\tau_{\gamma+1}(a)=0$, then $\tau_{\gamma} h_{\gamma+1}(a)=0=h_{\gamma+1}(a)$. This means $h_{\gamma+1}(a)=0$, that is $a \in \operatorname{Ker} h_{\gamma+1}$. Hence $a=0$, since $h_{\gamma+1}(a)=a$ for $a \in \operatorname{Ker} h_{\gamma+1}$. Thus $\tau_{\beta}$ is a monomorphism.

Case (ii) $\beta$ is a limit ordinal. If $\tau_{\beta}(a)=0$, then $\tau_{\alpha}(a \mid \alpha)=0$ for all $\alpha<\beta$. But $\tau_{\alpha}$ is a monomorphism for each $\alpha<\beta$, so $a \mid \alpha=0$ which by the sheaf properties implies that $a=0$. Thus the morphism $\tau: A \rightarrow C$ is indeed a monomorphism.

Finally we want to show that $C$ is an essential extension of $A$, and so consider $0 \neq D \subseteq C$. Since $\mathcal{L}$ is well-ordered we can find a smallest $\alpha \in \mathcal{L}$ such that $D_{\alpha} \neq 0$. Then $\alpha$ is not a limit ordinal, since otherwise we get a contradiction $0 \neq D_{\alpha} \rightarrow$ $\prod_{\gamma<\alpha} D_{\gamma}=0$. For $\delta$ such that $\alpha=\delta+1$, we have a commutative diagram

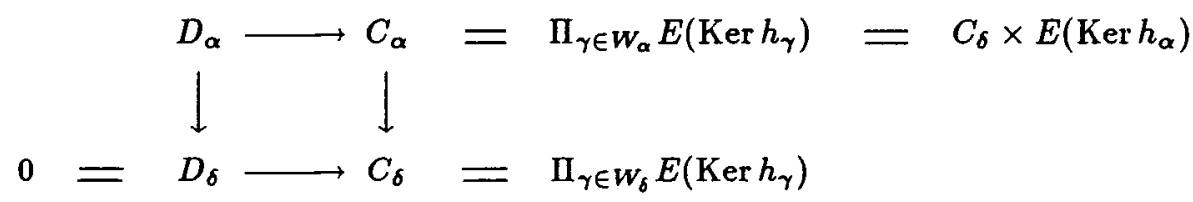

where the horizontal arrows are inclusions, and so we conclude that $D_{\alpha}=0 \times$ $D_{\alpha}^{-}$where $D_{\alpha}^{-} \subseteq E\left(\operatorname{Ker} h_{\alpha}\right)$. Hence there exists $0 \neq x \in \operatorname{Ker} h_{\alpha}$ such that $(0, x) \in D_{\alpha}$. Now $\operatorname{Im} \tau_{\alpha}=\operatorname{Im}\left(\tau_{\alpha-1} h_{\alpha} \prod \bar{h}_{\alpha}\right)$ and $(0, x) \in D_{\alpha}$ where $x \in \operatorname{Ker} h_{\alpha}$ implies $(0, x)$ belongs to $\operatorname{Im}\left(\tau_{\alpha-1} h_{\alpha} \prod h_{\alpha}\right)=\operatorname{Im} \tau_{\alpha}$. Hence $\tau_{\alpha}\left(A_{\alpha}\right) \cap D_{\alpha} \neq 0$ which means $\tau(A) \cap D \neq 0$. Thus $\tau: A \rightarrow C$ is an essential monomorphism. Also $C$ is an injective group and therefore $C$ is the injective hull of $A$.

Applied to the special case $\mathcal{L}=3$, Proposition 2.3 leads to the following. 


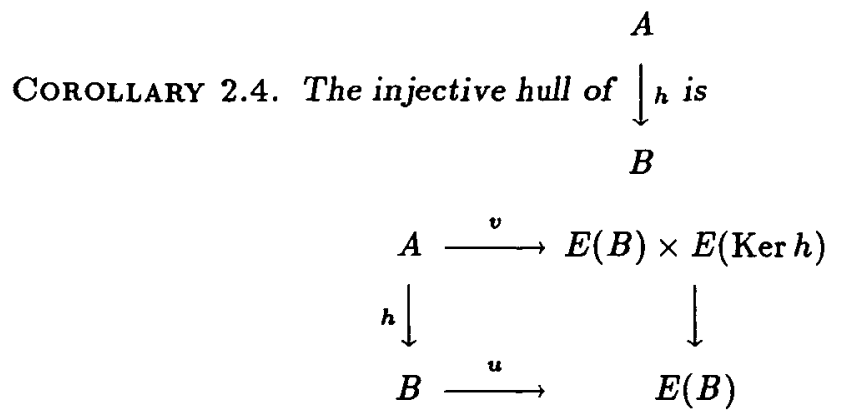

where $u$ embeds $B$ into its injective hull and $v=(u h) \prod k$ and $k: A \rightarrow E(\operatorname{Ker} h)$ extends the natural embedding $\operatorname{Ker}(h) \rightarrow A$.

LEMMA 2.5. For a boolean locale $\mathcal{L}$, if $E$ is not compact, then there exists $A, B \in$ $A b S h \mathcal{L}$ such that $A \subseteq B$ is essential but $A E \subseteq B E$ is not essential in $A b$.

Proof: Let $E=\bigvee_{i \in I} U_{i}$ where $I$ is an infinite set. Then there exists a countable subset, say $J$ of $I$, so that we can write $E=\left(\bigvee_{i \in J} U_{i}\right) \bigvee S$ where $S=\left(\bigvee_{i \in J} U_{i}\right)^{\prime}$. Since $\mathcal{C}$ is boolean, we can find a sequence $\left\{U_{n}\right\}_{n \in \omega}$ such that $U_{n} \wedge U_{m}=0$ and $E=\bigvee_{n \in \omega} U_{n}$. Define $A_{n}, B_{n} \in A b S h \downarrow U_{n}$ by $A_{n} U \doteq Z / p_{n} Z, B_{n} U \doteq Z / p_{n}^{2} Z$ for some prime $p_{n}$ where $p_{n} \neq p_{m}$ if $n \neq m$. Then $B_{n} \supseteq Z_{n}$ is essential in AbSh $\downarrow U_{n}$, for if $0 \neq \phi \in B_{n} U$ but $\notin A_{n} U$ then $\phi(a) \neq 0$ for some $a \in Z /\left(p_{n}^{2}\right)$ where order $a=p_{n}^{2}$ and so $0 \neq p_{n} \phi \mid \phi(a) \in A(\phi(a))$ which shows $A_{n} \subseteq B_{n}$ is essential. If $A, B \in A b S h \mathcal{L}$ are defined by $A=\prod_{n \in \omega}\left(\alpha_{n}\right)_{*} A_{n}, B=\prod_{n \in \omega}\left(\alpha_{n}\right)_{*} B_{n}$, where $\left(\alpha_{n}\right)_{*}: A b S h \downarrow U_{n} \rightarrow$ $A b S h \mathcal{L}$ corresponds to the morphism $\alpha_{n}: \mathcal{L} \rightarrow \downarrow U_{n}, U \rightsquigarrow U \wedge U_{n}(0.7)$, then $A U=$ $\prod_{n \in \omega} A_{n}\left(U \wedge U_{n}\right)$ and $B U=\prod_{n \in \omega} B_{n}\left(U \wedge U_{n}\right), U \in \mathcal{L}$. We claim $A \subseteq B$ is essential in $A b S h \mathcal{L}$. If $0 \neq \phi=\left(\phi_{n}\right) \in B U$, then for some $m \in \omega, 0 \neq \phi_{m} \in B_{m}\left(U \wedge U_{m}\right)$, and so by the above argument for some $a \in Z /\left(p_{m}^{2}\right), p_{m} \phi_{m} \mid \phi_{m}(a) \in A_{m}\left(\phi_{m}(a)\right)$. Since $U_{m} \wedge U_{n}=0$ for all $n \neq m$, we get $p_{m} \phi \mid \phi_{m}(a)=\left(p_{m} \phi_{n} \mid \phi_{m}(a)\right)_{n \in \omega} \in A U$, since all components are zero except when $n=m$. Hence $A \subseteq B$ is essential. To show that $A E \subseteq B E$ is not essential, consider $\phi=\left(\phi_{n}\right) \in B E=\prod_{n \in \omega} B_{n} U_{n}$ where $\phi_{n}$ is of order $p_{n}^{2}$. If $A E \subseteq B E$ was essential then there exists $k \in Z$ such that $k \phi_{n} \in A_{n} U_{n}$ for all $n \in \omega$. This means $p_{n} \mid k$ for all $n \in \omega$, hence $k=0$. Hence result.

LEMMA 2.6. The global functor $\Gamma: A b S h \mathcal{L} \rightarrow A b$ preserves injective hulls if and only if it preserves essential extensions.

Proof: $(\Leftarrow)$ Clear, by the hypothesis and the fact that the functor $\Gamma$ preserves injectives (since the functor $-\mathcal{C}$ is an exact left adjoint of $\Gamma(0.7)$ ).

( $\Rightarrow$ ) Let $A \mapsto B$ be an essential monomorphism in $A b S h \mathcal{L}$, and $A \mapsto A^{\prime}$ be the natural embedding of $A$ into its injective hull $A^{\prime}$. Then there exists $f: B \rightarrow A^{\prime}$ such that $f i=j$, which is actually a monomorphism (since $i$ is essential). By hypothesis 
$A E \longmapsto A^{\prime} E=A E \longrightarrow B E \longrightarrow A^{\prime} E$ is an essential monomorphism in $A b$, hence $i_{E}: A E \longmapsto B E$ is an essential monomorphism.

Proposition 2.7. The functor $\Gamma: A b S h \mathcal{L} \rightarrow A b$ preserves injective hulls if and only if $\mathcal{L}$ is a finite boolean locale.

Proof: $(\Leftrightarrow)$ By Lemma 2.6, it is enough to show that $\Gamma$ preserves essential extensions. If $\mathcal{L}$ is finite boolean then $\mathcal{L} \cong \mathcal{O}(X)$ for a finite discrete space $X$, therefore $A b S h \mathcal{L} \cong A b^{|X|}$. So $A \subseteq B$ essential in $A b S h \mathcal{L}$ implies $A\{x\} \subseteq B\{x\}$ is essential in $A b$ for all $x \in|X|$. Therefore $\prod_{x \in|X|} A\{x\}=\Gamma A \subseteq \Gamma B=\prod_{x \in|X|} B\{x\}$ is essential in $A b$, since finite product in $A b$ preserve essential extension.

$(\Rightarrow)$ By Lemma 2.6 it follows that the functor $\Gamma: A b S h \mathcal{L} \rightarrow A b$ preserves essential extensions. We first show that $\mathcal{L}$ is boolean. If not, then there exists a $W \in \mathcal{L}$ such that $W$ is dense. Let $A U \subseteq Q_{\mathcal{L}} U$ be the subgroup consisting of all $\phi \in Q_{\mathcal{L}} U$ such that $\bigvee_{0 \neq a \in Q} \phi(a) \leqslant U \wedge W$. Then $A$ is a subgroup of $Q_{\mathcal{C}}$ [2]. Define $B$ in $A b S h \mathcal{L}$ by $B U=A(U \wedge W)$, with the restrictions as given by $A$. Then $h: A \rightarrow B$ given by the restriction map of $A$ is a monomorphism, since $W$ is dense in $\mathcal{L}$. Moreover this monomorphism is essential for if $0 \neq \phi \in B U=A(U \wedge W)$ then clearly $\phi \mid$ $(U \wedge W)=h_{U \wedge W}(\phi)=\phi \neq 0$. By hypothesis $A E \rightarrow B E=A W=Q_{\mathcal{L}} W$ is an essential monomorphism. Consider $\phi \in Q_{\mathcal{C}} W$ with $\phi(1)=W$. By essentialness, there exists a $\psi$ in $A E$ such that $0 \neq h_{E}(\psi)=\psi \mid W=m \phi$ for some $m \in Z$. Then $(m \phi)(m)=W$, so $\psi(m) \wedge W=W$, that is, $W \leqslant \psi(m)$, which means $\psi(m)$ is dense in $\mathcal{L}$. So if $k \neq m$ then $\psi(m) \wedge \psi(k)=0$ implies $\psi(k)=0$, therefore $\psi(m)=E$. But $\psi \in A E$, so $\bigvee_{k \neq 0} \psi(k) \leqslant W$, and $m \neq 0$ implies $\psi(m) \leqslant W$, that is $E=W$, hence $\mathcal{L}$ is boolean. By Lemma 2.5 it follows that $E$ is compact. But $\mathcal{L}$ boolean implies each $U \in \mathcal{L}$ is compact, hence $\mathcal{L}$ is spatial. Therefore $\mathcal{L}=\mathcal{O}(X)$ for some discrete space $X$, which by compactness of $E$ means $X$ is a finite discrete space. Hence we have the result.

REMARK 2.8: If $\mathcal{L}$ is finite boolean then so are all $\downarrow U$, and hence all functors $\Gamma_{U}$ preserve injective hulls whenever $\Gamma=\Gamma_{E}$ does.

\section{ChaRACTERISING INJECTIVES For SOME SPECIAL Locales}

We have seen in our previous discussion that an injective $A \in A b S h \mathcal{L}$ has the following two properties:

(a) For all $U \in \mathcal{L}$, each $A U$ is an injective abelian group in $A b$.

(b) Whenever $V \leqslant U$ in $\mathcal{L}$, then the restriction $A U \rightarrow A V$ is a split epimorphism in $A b$.

Hence it is reasonable to ask if the properties (a) and (b) characterise injectives in $A b S h \mathcal{L}$. The answer is yes for some special locales which we shall discuss although the 
question still remains open for an arbitary $\mathcal{L}$. Recall that for $\mathcal{L}=3$, Banaschewski has shown that injectives in $A b S h 3$ are exactly those groups which satisfy the conditions (a) and (b) [2]. This fact is crucial in the following proofs.

Proposition 3.1. If $\mathcal{L}$ satisfies the descending chain condition then $A \in A b S h \mathcal{L}$ is injective if and only if it satisfies conditions (a) and (b).

Proof: To prove the remaining implication, consider any essential extension $B \supseteq$ $A$. If $A \subset B$, then $\mathcal{L}$ has $D C C$, we can find a minimal $S \in \mathcal{L}$ such that $A S \subset B S$. Clearly, for all $U<S, A U=B U$. If $W=\bigvee U(U<S)$ then $A W=B W$, since for any $b \in B W, b \mid U \in B U=A U$ for $U<S$ implies $b \in A W$, hence $W<S$.

Consider the commutative diagram,

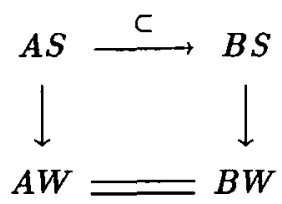

in $A b S h 3$. If $0 \neq b \in B S$, then by essentialness there exist $V \leqslant S$, and $m \in Z$ such that $0 \neq m b \mid V \in A V$. Now either $V=S$ which means $0 \neq m b \in A S$, or $V<S$ and then $V \leqslant W$ so $0 \neq m b|V=(m b \mid W)| V$ implies $0 \neq m b \mid W \in B W=A W$. Thus $B S \rightarrow B W$ is an essential extension of $A S \rightarrow A W$ in $A b S h 3$. But by the given hypothesis $A S \rightarrow A W$ is injective in $A b S h 3$ [2] and hence $A S=B S$. Thus $A=B$, which means $A$ is injective in $A b S h \mathcal{L}$.

Corollary 3.2. If $\mathcal{L}$ is finite or well-ordered then the conditions (a) and (b) characterise injectives in $A b S h \mathcal{L}$.

Proof: Clear, since these locales have descending chain conditions.

Proposition 3.3. For any inversely well-ordered $\mathcal{L}, A \in A b S h \mathcal{L}$ is injective if and only if it satisfies conditions (a) and (b).

PROOF: If $\mathcal{L}$ is inversely well-ordered then the elements of $\mathcal{C}$ may be arranged in the form $E=U_{0}>U_{1}>U_{2} \cdots>U_{\lambda}=0$, so that $L^{\text {opp }} \cong \lambda+1$ for some ordinal $\lambda$. Since each non-empty subset of $\mathcal{L}$ has a largest element it follows that every element in $\mathcal{L}$ has only trivial covers, hence every presheaf on $\mathcal{L}$ is also a sheaf on $\mathcal{L}$. In particular, $Z_{\mathcal{L}} U_{\alpha}=Z$ for all $\alpha$. If $A \in A b S h \mathcal{L}$ satisfies conditions (a) and (b), then we claim that $A$ is injective. The proof will use the Baer criterion (1.1), so consider a diagram,

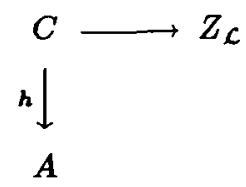


where the horizontal arrow is the inclusion. Our aim is to extend $h$ to all of $Z_{\mathcal{L}}$. If $C=0$, then we are done. If $C \neq 0$, then we can pick the first $\alpha_{0}$ such that $C U_{\alpha_{0}} \neq 0$. If $U_{\alpha}>U_{\beta}$, then the commutativity of the diagram

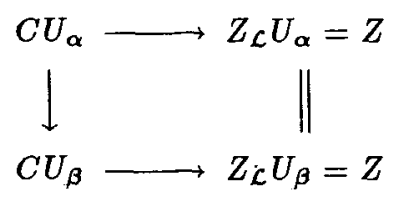

where the horizontal arrows are inclusions, implies $C U_{\alpha} \subseteq C U_{\beta}$. Let $U_{\alpha_{1}}$ be the first element in $\mathcal{L}$ such that $C U_{\alpha_{0}} \subset C U_{\alpha_{1}}$. Proceeding in the same fashion we obtain a strictly ascending chain of subgroups of $Z$ given by $0 \neq C U_{\alpha_{0}} \subset C U_{\alpha_{1}} \subset C U_{\alpha_{2}} \subset \ldots$. Since $Z$ is noetherian, this chain must terminate after a finite number of steps and so for some $n, C U_{\alpha_{n}}=C U_{\alpha}$ for all $\alpha \geqslant \alpha_{n}$.

If we consider the finite chain $F=U_{\alpha_{0}}>U_{\alpha_{1}}>\cdots>U_{\alpha_{n}}$ (which has only trivial covers), then the presheaf $A U_{\alpha_{0}} \rightarrow A U_{\alpha_{1}} \rightarrow \cdots \rightarrow A U_{\alpha_{n}}$ satisfies condition (a) and (b) and so by our last result it is an injective group in $A b S h F$. Hence there exist morphisms $g_{U_{\alpha_{i}}}: Z \rightarrow A U_{\alpha_{i}}$, such that $g_{U_{\alpha_{i}}} \mid C_{U_{\alpha_{i}}}=h_{U \alpha_{i}}$, and $g_{U_{\alpha_{i}+1}}=g_{U_{\alpha_{i}}} \mid U_{\alpha_{i+1}}$ for all $i=0,1, \ldots, n$. For any $U_{\alpha} \in \mathcal{L}$, where $\alpha \neq \alpha_{0}, \alpha_{1}, \ldots, \alpha_{n}$ define $g_{U_{\alpha}}$ as follows: $g_{U_{\alpha}}=i_{\alpha} g_{U_{\alpha_{0}}}$ if $0 \leqslant \alpha \leqslant \alpha_{0}$ where $i_{\alpha}: A U_{\alpha_{0}} \rightarrow A U_{\alpha}$ is the inclusion into the product $A U_{\alpha_{0}} \rightarrow A U_{0}$ followed by the restriction may $A U_{0} \rightarrow A U_{\alpha}$

$$
\begin{array}{cl}
g_{U_{\alpha}}=g_{U_{\alpha_{0}}} \mid U_{\alpha} & \text { if } \alpha_{0} \leqslant \alpha<\alpha_{1} \\
g_{U_{\alpha}}=g_{U_{\alpha_{1}}} \mid U_{\alpha} & \text { if } \alpha_{1} \leqslant \alpha<\alpha_{2} \\
\vdots & \\
g_{U_{\alpha}}=g_{U_{\alpha_{n}}} \mid U_{\alpha} & \text { if } \alpha \geqslant \alpha_{n}
\end{array}
$$

Since $g_{U_{\alpha_{i+1}}}=g_{U_{\alpha_{i}}} \mid U_{\alpha_{i+1}}$, it follows for all $U_{\alpha} \leqslant U_{\alpha_{0}}$, we have $g_{U_{\alpha}}=g_{U_{\alpha_{0}}} \mid U_{\alpha}$. It remains to show that $g$ extends $h$. Let $U \alpha, U_{\beta}$ be arbitary elements of $\mathcal{L}$ such that $U_{\alpha} \leqslant U_{\beta}$. Then there are three cases:
(i) $U_{\alpha_{0}} \geqslant U_{\beta} \geqslant U_{\alpha}$
(ii) $U_{\beta} \geqslant U_{\alpha_{0}} \geqslant U_{\alpha}$
(iii) $U_{\beta} \geqslant U_{\alpha} \geqslant U_{\alpha_{0}}$.

Case (i). In this case $g_{U_{\beta}}=g_{U_{\alpha_{0}}} \mid U_{\beta}$, so 
$g_{U_{\beta}}\left|U_{\alpha}=\left(g_{U_{\alpha_{0}}} \mid U_{\beta}\right)\right| U_{\alpha}=g_{U_{\alpha_{0}}} \mid U_{\alpha}=g_{U_{\alpha}}$ hence the diagram

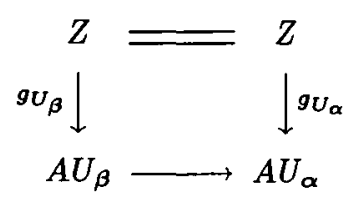

commutes.

Case (ii). $g_{U_{\alpha}}=i_{\beta} g_{U_{\alpha_{0}}}$ hence

$$
g_{U_{\beta}}\left|U_{\alpha}=\left(g_{U_{\beta}} \mid U_{\alpha_{0}}\right)\right| U_{\alpha}=\left(\left(i_{\beta} g_{U_{\alpha_{0}}}\right) \mid U_{\alpha_{0}}\right)\left|U_{\alpha}=g_{U_{\alpha_{0}}}\right| U_{\alpha}=g_{U_{\alpha}}
$$

Case (iii). $g_{U_{\beta}}=i_{\beta} g_{U_{\alpha_{0}}}$, therefore

$$
g_{U_{\beta}}\left|U_{\alpha}=\left(i_{\beta} g_{U_{\alpha_{0}}}\right)\right| U_{\alpha}=i_{\alpha} g_{U_{\alpha_{0}}}=g_{U_{\alpha}}
$$

Hence, we conclude that $g$ is indeed a morphism of sheaves. Now to check that $g$ extends $h$, we consider any $U_{\alpha} \in \mathcal{L}$. If $U_{\alpha}>U_{\alpha_{0}}$, then $C U_{\alpha}=0$, so $g_{U_{\alpha}} \mid C_{U_{\alpha}}=0=h_{U_{\alpha}}$. So let us suppose that $U_{\alpha_{0}} \geqslant U_{\alpha}$. Then $g_{U_{\alpha}}\left|C U_{\alpha}=g_{U_{\alpha}}\right|$ $C U_{\alpha_{0}}\left(\right.$ if $\left.C U_{\alpha}=C U_{\alpha_{0}}\right)=\left(g_{U_{\alpha_{0}}} \mid C U_{\alpha_{0}}\right)\left|U_{\alpha}=h_{U_{\alpha_{0}}}\right| U_{\alpha}=h_{U_{\alpha}}$. If $C U_{\alpha} \neq C U_{\alpha_{0}}$, then $\alpha_{1} \leqslant \alpha$. If $C U_{\alpha}=C U_{\alpha_{1}}$, then again we are done by the same argument with $\alpha_{1}$ in place of $\alpha_{0}$ since $g_{U_{\alpha_{1}}} \mid C U_{\alpha_{1}}=h_{U_{\alpha_{1}}}$. Otherwise, $C U \alpha \supset C U_{\alpha_{1}}$ and in that case $\alpha_{2} \leqslant \alpha$ and one can proceed as before. Continuing in the same way one sees that $g_{U_{\alpha}} \mid C U_{\alpha}=h_{U_{\alpha}}$ for all $\alpha$, that is $g$ extends $h$. This shows $A$ is injective.

Corollary 3.4. In $A b S h \mathcal{L}$ where $\mathcal{L}$ is inversely well-ordered, the direct sum of injectives is injective.

Proof: Let $A=\oplus_{i \in I} A_{i}$, where $A_{i}$ is an injective group in $A b S h \mathcal{L}$. Then each $A_{i} U$ is divisible in $A b$, for all $U \in \mathcal{L}$. Therefore $A U=\oplus A_{i} U$ is divisible in $A b$. For any $V \leqslant U$ in $\mathcal{L}$, each $A_{i} U \rightarrow A_{i} V$ is a split epimorphism, $\oplus_{I} A_{i} U \rightarrow \oplus_{I} A_{i} V$, that is $A U \rightarrow A V$ is a split epimorphism in $A b$. By Proposition 3.3,A is injective, hence the result.

COUNTEREXAMPLE 3.5: Here we show that the direct sum of injectives in $A b S h \mathcal{L}$ is not always injective for an arbitary $\mathcal{L}$. Consider an infinite space $X$, with the topology given by $U \in \mathcal{O} X$ if and only if $U=X, \emptyset$, or $x \notin U$ where $x$ is a fixed point. of $X$.

Then $\{y\} \in \mathcal{O} X$ if and only if $y \neq x$. For all $z \in|X|$, define $A_{z}=\phi_{*}(Q)$ where $\phi: \mathcal{C} \rightarrow 2$ is the locale lattice homomorphism corresponding to the point $z \in|X|(0.7)$. Then $A_{z} U \subseteq B: U \rightarrow Q^{U}$ consists of all $a \in B U$, with support contained in $\{z\}$. Let 
$A=\oplus_{z \in|X|} A_{z}$. We claim $A$ is not injective, although each $A_{z}$ is an injective group (0.7). Note that $A$ can be taken as a subgroup of $B$, and $f \in B X$ belongs to $A X$ if and only if there exists a cover $X=\bigcup_{i \in I} U_{i}$ such that $f \mid U_{i}$ is of finite support for all $i \in I$. Since $X$ has only trivial covers it follows that $X=U_{i}$ for some $i \in I$. Hence $A X$ consists of all $f$ in $Q^{X}$ of finite support and so $A X \subset B X$. Note that for $X \neq U, A U=B U$. Now let $0 \neq a \in B X$. If $a(y) \neq 0$ for any $y \neq x$ then $0 \neq a \mid\{y\} \in A\{y\}$, and otherwise $a(y)=0$ for all $y \neq x$ so that $a \in A X$. Hence $B$ is an essential extension of $A$ and therefore $A$ is not injective. Since $B$ is also injective (0.7), if follows that $B$ is the injective hull of $A$.

\section{REFERENCES}

[1] R. Balbes and P. Dwinger, 'Distributive lattices' (University of Missouri Press 1975).

[2] B. Banaschewski, 'When are divisible abelian groups injective?', Quaestiones Math. 4 (1981), 285-307.

[3] B. Banaschewski, 'Injective sheaves of Abelian groups: a counterexample', Canad. J. Math. 32 (1980), 1518-1521.

[4] B. Banaschewski, 'Coherent Frames', Lecture notes in Math. 871.

[5] B. Banaschewski, 'Recovering a space from its abelain Sheaves': Seminar talks, (McMaster University September 1980).

[0] M. Ebrahimi, 'Algebra in a topos of sheaves' (Doctoral dissertation McMaster University 1980).

[7] M.P. Fourman and D.S. Scott, 'Sheaves and logic', in Application of sheaves Proceedings, Durham 1977: Lecture Notes in Math. (Springer Verlag, Berlin Heidelberg New York 1979).

[8] P. Freyd, 'Abelian Categories' (Harper and Row Publishers 1964).

[9] L. Fuchs, 'Infinite abelian groups' (Academic press, Vol. 1 1970).

[10] R. Godement, 'Theorie des Fisceaux' (Hermann, Paris 1958).

[11] R. Harting, 'A remark on injective sheaves of abelain groups' (to appear).

[12] P.T. Johnstone, 'Topos Theory' (Academic Press, London 1977).

[13] P.T. Johnstone, 'Stone spaces': Cambridge studies in Advanced Mathematics 3 (Cambridge Univeristy Press, Cambridge 1982).

[14] I. Kaplansky, 'Infinite abelain groups' (University of Michigan Press, Ann Arbor, Michigan 1968).

[15] S. MacLane, 'Categories for the working mathematician' 5: Graduate text in Mathematics (Springer Verlag 1971).

[18] N. Popenscu, 'Abelian categories with applications to rings and modules' (Academic Press 1973).

The Catholic University of America

Washington, D.C.

United States of America. 